the bronchi, the stomach, or in some obscure nervous disturbance. It is a question of paramount importance, more especially in relation to that intractable affection known as hay asthma and megrim or sick headache, and one of equal interest to the general practitioner and to the specialist. 'The etiology of these disorders being elucidated, enables us to apply a rational treatment with more probability of cure than the heretofore empirical methods could give us. The good results of the above observations in treatment have been demonstrated by the number of recorded cures.

Cases of obstinate irritative cough, more especially annoying at night, have been cured by simply ridding the patient of a slight chronic rhinitis, which caused the cough by the irritation of retained secretion on sensitive spots of the nasal mucous membrane. You are all familiar with the efficacy of the old nursery remeely of a drink of water for a child's cough at night, which is explained by its ridding the posterior nares and pharynx of any secretion that might have accumulated and which was the irritating cause; and also in all probability there can be found sensitire spots in the pharynx, the irritation of which will cause retlex phenomena. The record of the cases of reflected neuroses of the nose, includes besides cough, megrim or sick headache, cephalalgia, tinnitus aurum and giddiness unaccompanied by deafness, otalgia, certain apparent disturbances of the circulation, such as slight and recurring odema of the face and eyelids, asthmatic attacks, and that annoying group of symptoms known as hay fever, hay asthma, rose cold, etc., etc., all of which have been shown to be caused by pathological nasal changes and cured by their removal. I have also met a case of recurring conjunctivitis of same causation. Any treatment therefore of these affections, which, in the face of these investigations and clinical facts, would leave the possibility of nasal causation, by reflected irritation out of the question, would be, to say the least of it, highly unscientific. But in tracing the nasal relation, the question arises, as to what symptoms about the nasal mucosa we are to look for to give us any positive ground to assume that we are dealing with a reflex neurosis of the nose. I doubt if in the present state of our knowledge, this question can be definitely answered.

In many cases there would be frequent sneezing, a watery discharge from the nose, and occasional and intermittent stoppage of one or both nostrils, possibly some slight and fleeting redema of the face; in others these might be wholly or in part absent.

But still, it is all-important to have a proper nasal examination made, more especially in the absence of any other defined causation. A careful examination of the nose might reveal swelling of the turbinated corpora cavernosa; or the use of the probe would show the sensitive spots by the irritation caused from contact, giving rise to sneezing, asthmatic breathing, neuralgic pain, notably toothache, possibly gastric disturbances, or sometimes one-sided perspiration, especially of the extremities.

In hay fever, however, we do not need these guides to a diagnosis, nor can there be any doubt because we are all so familiar with the group of symptoms known by that name, and known heretofore as the disgrace of the Rhinologist as trachoma is of the ophthalmologist. Hence the proof that it is a reflex phenomenon of certain intra-nasal changes, the special irritation of the sensitive spots or structures being set up by various causes, whether pollen or other agents, whether periodically or at irregular times, is a great advance in the right direction. Possibly Dr. Mackenzie's theory of an abnormal excitability (inherited or acquired) of the reflex nerve centres being the essential principle of the reflex action instead of excitability of the terminal fibres of the nerves (vaso motor, or vaso dilator) is more acceptable, at least to my mind, whilst not agreeing with him in the necessity of engorgement or swelling of the turbinated tissues in order to induce reflex phenomena. I think that this idiosyncratic excitability of the reflex nerve centres explains better the different forms this reflex neurosis takes in different individuals from irritation of the same or corresponding sensitive spots in the nasal mucosa. But here as elsewhere, when dealing with the sympathetic and its functions we are wandering in a maze of uncertainty and speculation that belongs rather to experimental physiology. 'The treatment should be directed to diminishing the tendency to irritation of the sensitive area or spots on the nasal mucosa by both internal and local treatment. Failing in this the sensitive areas, which must be carefully localized with the probe, should be destroyed by caustic agents or by the galvano-cautery. Iatterly much has been written about the use of the galvanocautery, and probably as a result of this its use is likely to run into abuse. It is, undoubtedly, a very valuable agent in the treatment of nasal affections, but we should be careful in selecting appropriate cases for its application. Whilst considerable success has attended this line of treatment in the reflex neuroses, still relapses occur, and make us look forward to the further light on the subject that greater experihnce will give.

\section{ADDRESS IN DENTAL AND ORAL SURGERY.}

DELIVERED IN GENERAL SESSION AT THE THIRTYSIXTH ANNUAI, MEETING OF THE AMERICAN MEDICAL ASSOCIATION.

BY WALTER W. ALLPORT, M. D.

OF CHICAGO, ILL.

The introduction of cocaine as a local anasthetic, and the more general use of peroxide of hydrogen $\left(\mathrm{H}^{2} \mathrm{O}^{2}\right)$ in the treatment of dental and oral diseases, are the principal advances made in the medical department of this practice during the year for which this report is made.

The two forms of cocaine which have been most generally used in surgery are the hydrochlorate and the oleate. In operations in the mouth involving the mucous membranes, together with the immediately subjacent tissues, these preparations of the drug have proven so efficient that there is little ques- 
tion as to its value as a local anæsthetic in such to itself, and sometimes even after the tooth is excases. But its action upon deeper structures, such tracted, will point through the external tissues at the as involve the roots of teeth, is so uncertain as to lower margin of the jaw, and occasionally downwards render its practical benefits questionable in the op- between the muscles of the neck and open at various eration of extraction. In the surgical treatment of points, even as low down as the clavicle. The usual pocketscaused by pyorrhea alveolaris, the anæsthetic treatment in such cases, is to extract the tooth and effect of this agent is often so great as to render this sometimes very painful operation comparatively painless, and its employment in such cases should rarely be dispensed with. In the treatment of hypersensitive dentine as well as in the removal of toothpulps, its action as an anæsthetic has, under some circumstances, seemed to be all that could be desired. But in far the greater number of cases it has proved of little practical value. More recently however, a new form of cocaine, known as the citrate, has been introduced in Germany by Merck, and is now being manufactured by McKesson and Robbins, of New York. In a series of experiments, conducted by Dr. John S. Marshall, of Chicago, it has been shown that for operations upon sub-mucous tissues or in the extraction of teeth, it seems to possess no special advantages over the preparations previously named. But when applied to dentine or the pulp, its action -although not always positive-seems to be more reliable, especially upon the dentine, and gives promise of better results. Under favorable conditions it produces anæsthesia of the parts in from five to ten minutes, and the duration of the effect is of sufficient length to afford time for the preparation of the cavity. 'This effect has, in some cases, been prolonged for more than hour. 'The pulp has been extirpated without pain after the drug has been applied in from three to twelve minutes.

If the citrate of cocaine be kept in solution for more than three or four days, it decomposes and loses its active properties. As introduced by $\mathrm{Mr}$. Merck for dental purposes, it is made into pills by incorporating it with gum tragicanth dissolved in glycerine, each pill containing $1 / 8$ grain of the citrate. In this form it keeps well. A pill is applied to the sensitive cavity and covered with a cotton pledget, moistened in tepid water. It should be allowed to remain from five to twelve minutes, when-if at allthe desired result is produced. In twenty per cent of the cases where this remedy has been employed, it has proven unsuccessful, but it is hoped that this percentage will be reduced by a better knowledge of the drug and the improved methods of its preparation and use.

With this end in view and at the suggestion of Dr. Marshall, McKesson and Robbins are now manufacturing granules containing one-sixteenth of a grain of the citrate of cocaine, without glycerine or any other saccharine excipient, so that the obtundent may act more promptly than it can in the presence of sugar.

The peroxide of hydrogen, $\left(\mathrm{H}^{2} \mathrm{O}^{2}\right)$, although not a new remedy, has only within the last few years gained much prominence in the treatment of surgical diseases. One of its uses in dental and oral surgery, is in blind or deep-seated abcesses, such as arise from roots of diseased teeth. As the tendency of pus is always downwards, when these cases occur in the lower jaw, it is not infrequent that the abcess, if left evacuate the pus through the alveolus, but it often happens that the formation of pus and the continuance of suppuration is not checked, and the abscess points, or is opened through the external tissue of the face or neck, leaving, when healed, a disfiguring scar.

By injecting peroxide of hydrogen into such abscesses before they point through the external tissues, this serious disfigurement can usually be averted, and the suppurative process is materially shortened. It is also a valuable aid for the evacuation of the purulent contents of the antrum of Highmore, in catarrhal and suppurative inflammations, and especially so where the sinuses are divided into two or more pockets by bony septi. These cases are often protracted by the inability of the surgeon to perfectly evacuate them. But with this preparation it becomes a simple matter, after access has been gained to the cavity by the extraction of a tooth or the perforation of its external wall in the proper place at the juncture of the cheek with the alveolar border. A free opening must always be made for the escape of the contents, in order to avoid pressure from the rapid evolution of gas. Two or three applications of a drachm each is usually sufficient to completely empty the sac.

It is used with most gratifying results in the treatment of pyorrhea alveolaris, and is an invaluable agent in treating pulpless teeth, as by its action, all decomposed matter from the pulp chamber and dentinal tubuli is readily ejected; thereby removing the most fruitful causes of the discoloring of this class of teeth, of inflammation of the periclental membrane, as well as alveolar abscesses.

The efficacy of peroxide of hydrogen depends upon the ease with which it is decomposed into oxygen and water. Pus is one of the many substances which causes this decomposition. Hydrogen peroxide acts first, chemically, and then mechanically. When the decomposition takes place the oxygen is set free and escapes from a licpuid to a gaseous form; this expansion of the gas distends the pus cavity, and as it escapes from the orifice, it carries much of the pus with it, and its application should be repeated until all purulent accumulations are evacuated. The liberated oxygen, being in a nascent or active condition, rapidly oxydizes the products of suppuration and destroys many of the micro-organisms of suppuration'. Hence it is a disinfectant and anti-septic.

Finally, peroxide of hydrogen, after its decomposition, leaves no material in the system which is foreign to the system itself and it is therefore one of the most efficient and harmless disinfectants and antiseptics that can be used, in all forms of purulent inflammation.

\footnotetext{
1See Gradle on "Bacteria and the Germ Theory of Disease," pp. 39 and $15 x$.
} 